\title{
Complement depletion with cobra venom factor alleviates acute hepatic injury induced by ischemia-reperfusion
}

\author{
BING WANG, HUA XU, JIAN LI, HONG-MEI GAO, YING-HONG XING, ZHU LIN, \\ HONG-JIE LI, YONG-QIANG WANG and SHU-HUACAO
}

\begin{abstract}
Department of Intensive Care Unit and Key Laboratory for Critical Care Medicine of The Ministry of Health, Emergency Medicine Research Institute, Tianjin First Center Hospital, Tianjin 300192, P.R. China
\end{abstract}

Received March 2, 2018; Accepted August 28, 2018

DOI: $10.3892 / \mathrm{mmr} .2018 .9484$

\begin{abstract}
Increasing evidence has demonstrated that complement activation is required for ischemia-reperfusion injury (IRI)-induced hepatic damage, and cobra venom factor (CVF) can deplete the complement components. The aim of the current study was to investigate the effect and intrinsic mechanism of CVF pretreatment on IRI-induced acute hepatic injury in rats. Acute hepatic injury in rats was induced by bone fracture to simulate trauma, followed by hemorrhage for $90 \mathrm{~min}$, and then the rats were resuscitated for a period of $20 \mathrm{~min}$ of reperfusion. The survival times under different CVF treatment doses and schedules for rats with IRI were evaluated. Hepatic tissues and serum samples were analyzed for acute hepatic injury, complement activation, inflammatory mediator release and apoptosis at predetermined times and compared between the IRI group and the CVF pretreatment + IRI groups. Compared to the rats with IRI alone, the survival times were significantly improved among rats with IRI receiving a high-dose or low-dose CVF pretreatment (all $\mathrm{P}<0.01)$. Upon histological examination, severe hepatic damage was observed in the rats with IRI, accompanied by liver function deterioration, complement and membrane attack complex activation, inflammatory mediator release and hepatic cell apoptosis. CVF pretreatment significantly attenuated the hepatic injury through depletion of anaphylatoxic C5a and membrane attack complex C5b-9 activation, and subsequent inhibition of inflammatory mediator release and hepatic cell apoptosis (all $\mathrm{P}<0.05$ ). The results indicated that CVF pretreatment ameliorates IRI-induced acute hepatic injury. However, further studies are required to determine
\end{abstract}

Correspondence to: Dr Yong-Qiang Wang, Department of Intensive Care Unit and Key Laboratory for Critical Care Medicine of The Ministry of Health, Emergency Medicine Research Institute, Tianjin First Center Hospital, 24 Fukang Road, Nankai, Tianjin 300192, P.R. China

E-mail: wangyongqiang1100@163.com

Key words: cobra venom factor, acute hepatic injury, ischemia-reperfusion injury whether this therapy could be a potential agent for the treatment of IRI injuries in clinical settings.

\section{Introduction}

Ischemia-reperfusion injury (IRI) can induce acute hepatic injury, which is associated with a high level of mortality $(1,2)$. The liver is subjected to IRI in different medical conditions, including hepatic resection and liver transplantation (3). Notably, IRI is not only a pathophysiological process involving liver injury; it also affects multiple organs (4). However, the exact intrinsic mechanism of hepatic injury caused by IRI is still unknown.

Recent advances have demonstrated that complement activation is responsible for the pathogenesis of IRI-induced acute hepatic injury (5). The membrane attack complex C5b-9 is formed during complement pathway activation in IRI-induced acute hepatic injury, leading to lysis and destruction of target cells. Additionally, anaphylatoxins C3a and C5a are released and interact with their receptors, causing activation of the inflammatory and apoptotic pathways (6,7). Inhibition of abnormal complement activation potentially alleviates IRI-induced acute hepatic injury (8).

Cobra venom factor $(\mathrm{CVF})$ is a stable anticomplement protein from cobra venom. It is a structural and functional analog of the human complement component $\mathrm{C} 3 \mathrm{~b}$ (the active fragment of $\mathrm{C} 3$ ). Similar to C3b, CVF binds to factor B, which is then cleaved by factor $\mathrm{D}$ to form the bimolecular complex $\mathrm{CVF} / \mathrm{Bb}$; and $\mathrm{CVF} / \mathrm{Bb}$ is a $\mathrm{C} 3 / \mathrm{C} 5$ convertase that cleaves both complement components $\mathrm{C} 3$ and C5 (9-11). Therefore, CVF/Bb can continuously activate $\mathrm{C} 3$ and C5 to deplete complement components and inhibit its activation, achieving the desired anticomplement effect.

Several studies have demonstrated that CVF pretreatment can alleviate acute lung injury, myocardial injury and cerebral injury induced by IRI, and prolong the survival time following organ transplantation (12-15). The aim of the current study was to investigate the effect and mechanism of CVF pretreatment on IRI-induced acute hepatic injury in rats. The results indicated that CVF pretreatment significantly attenuated hepatic injury through depletion of completion activation and subsequent inhibition of inflammatory mediator release and hepatic cell apoptosis. However, further studies are required to determine whether this therapy may be a potential agent for the treatment of IRI injuries in clinical settings. 


\section{Materials and methods}

Establishment of the rat model of IRI-induced hepatic injury. All of the experiments were performed as approved by the Laboratory Animal Care Committee of Tianjin First Center Hospital (approval no. 2015013Z). One hundred and seventy six-week old male and female Sprague Dawley rats (200-250 g; 1:1 ratio) were obtained from the Experimental Animal Center, Academy of Military Medical Sciences (Beijing, China; Animal qualified batch number: SCXK-2007-004). These rats were housed in the rooms of the Laboratory Animal Care of Tianjin First Center Hospital with specified pathogen-free conditions. The room was maintained at room temperature under relative humidity $40-60 \%$. Food and water were provided ad libitum.

To simulate a clinical traumatic hemorrhagic shock situation, the rat model of IRI-induced acute hepatic injury was successfully established, as described previously (16). Briefly, rats were anesthetized by intraperitoneal injection of chloral hydrate $(200 \mathrm{mg} / \mathrm{kg})$, as described previously $(17,18)$. An open mid-diaphyseal transverse fracture was created in the left femur to induce trauma, after which a mean arterial blood pressure of $30 \pm 5 \mathrm{mmHg}$ was maintained for $90 \mathrm{~min}$ via hemorrhage at $2.5 \mathrm{ml} / 100 \mathrm{~g}$. Subsequently, the rats were resuscitated for a period of $20 \mathrm{~min}$ with Ringer's lactate solution at a constant rate to manage the shock. The volume of Ringer's solution was twice that of the blood loss. Finally, the catheters were removed, and the vessels were ligated.

Treatment design. The experimental rats were randomly and equally divided into five groups: i) The control group underwent the same anesthetic process without surgical procedures; and the following four groups all have acute hepatic injury was induced by bone fracture to simulate trauma, followed by hemorrhage, and then were resuscitated of reperfusion as described above: ii) the IRI group; iii) the CVF1 group received a high-dose $(100 \mu \mathrm{g} / \mathrm{kg})$ CVF (cat. no. C8406, Sigma-Aldrich; Merck KGaA, Darmstadt, Germany) pretreatment at $24 \mathrm{~h}$ before the surgery + IRI; iv) the CVF2 group received low-dose $(50 \mu \mathrm{g} / \mathrm{kg}) \mathrm{CVF}$ pretreatment at $24 \mathrm{~h}$ before the surgery + IRI; and v) the CVF3 group received low-dose $(50 \mu \mathrm{g} / \mathrm{kg})$ CVF at day 2 after IRI. The CVF frozen powder was dissolved in normal saline.

Survival time analyses. A total 170 rats were used for survival time assessment and the intrinsic mechanism determination. Firstly, the effect of CVF administration on survival time was assessed in the five groups described above ( $\mathrm{n}=10$ per group), and survival was defined as the time between the first treatment and the time of rat death or $48 \mathrm{~h}$ follow-up for censored rats. Subsequently, 120 rats were used for intrinsic mechanism determined among the control, IRI, and CVF2 + IRI groups. In each group, 10 rats were sacrificed at $1,3,6$, and $24 \mathrm{~h}$ after IRI, and the hepatic tissues and at least $4 \mathrm{ml}$ blood samples were collected and analyzed.

Histological analyses. For hematoxylin-eosin (H\&E) staining, the hepatic tissues were fixed with at least $15 \mathrm{ml} 10 \%$ neutral formaldehyde solution for $\geq 24 \mathrm{~h}$. Subsequently, the samples were dehydrated, embedded in paraffin, sectioned into $4-\mu \mathrm{m}$-thick slices, and mounted onto slides. Following heating at $60^{\circ} \mathrm{C}$ for $2 \mathrm{~h}$, slicing and dewaxing at room temperature for $10 \mathrm{~min}, \mathrm{H} \& \mathrm{E}$ staining was conducted at room temperature for $10 \mathrm{~min}$, and the slides were observed under an Olympus BX40F light microscope (Olympus Corporation, Tokyo, Japan).

For the immunohistochemical assay, the $4-\mu$ m-thick hepatic tissue sections were deparaffinized, rehydrated, and treated with $3 \% \mathrm{H}_{2} \mathrm{O}_{2}$ at $20^{\circ} \mathrm{C}$ for $10 \mathrm{~min}$. Antigen retrieval was performed by boiling the sections in Tris/EDTA Antigen Retrieval Solution (pH 8.0) for $2 \mathrm{~min}$. Nonspecific antibody binding was blocked using $3 \%$ bovine serum albumin at $20^{\circ} \mathrm{C}$ for $30 \mathrm{~min}$. Subsequently, the tissue sections were incubated with anti-C5b-9 antibody (cat. no. ab55811; 1:500), anti-Bcl-2 associated X, apoptosis regulator (Bax) antibody (cat. no. ab32503; 1:500), anti-Bcl-2 apoptosis regulator (Bcl-2) antibody (cat. no. ab59348; 1:500; all Abcam, Cambridge, UK), or control IgG overnight at $4^{\circ} \mathrm{C}$. The bound antibodies were detected with horseradish peroxidase (HRP)-conjugated secondary antibodies (cat. no. ab205718; 1:100; Abcam) at room temperature for $1 \mathrm{~h}$, and the products were visualized using a 3, 30'-diaminobenzidine tetrahydrochloride (DAB) staining kit (Tiangen Biotech Co., Ltd., Beijing, China) at room temperature for $2 \mathrm{~min}$ and counterstained with hematoxylin at room temperature for $5 \mathrm{~min}$. The specimens were examined with an inverted fluorescence microscope (IX50; Olympus Corporation, Tokyo, Japan).

In addition, the expression levels of C5b-9 deposition in specimens were assessed by two independent researchers who were double-blinded manner. Their conclusions were in complete agreement for $85 \%$ of specimens indicating this scoring method was highly reproducible. If two agreed with the scoring results, the value was selected. If there was a disagreement, then the third researcher worked collaboratively to confirm the score. For evaluation of C5b-9 deposition, a semi-quantitative scoring criterion was used, in which both staining intensity and positive areas were recorded from 0 to 100 .

ELISA. For the ELISA, the serum samples were centrifuged at $3,000 \mathrm{r} / \mathrm{min}(12,000 \mathrm{xg})$ for $5 \mathrm{~min}$ at $4^{\circ} \mathrm{C}$ and were stored at $-20^{\circ} \mathrm{C}$ until use. Alanine transaminase (ALT; cat. no. EIA-325), aspartate transaminase (AST; cat. no. EIA-326), total hemolytic complement (CH50; cat. no. EIA-121), C5b-9 (cat. no. EIA-765), tumor necrosis factor (TNF)- $\alpha$ (cat. no. SRTA00), interleukin (IL)-1 $\beta$ (cat. no. DY501) and C5a (cat. no. DY2150) were measured using the monoclonal anti-rat capture antibody (ZSGB-BIO, Inc., Beijing, China and R\&D Systems, Inc., Minneapolis, MN, USA), according to the manufacturer's instructions. The colorimetric reaction was measured using a Benchmark microplate reader (Benchmark Electronics, Inc., Scottsdale, AZ, USA).

Semi-quantitative reverse transcriptase-polymerase chain reaction $(R T-P C R)$. Guided by the manufacturer's instructions, total RNA was extracted using the TRIzol reagent (Thermo Fisher Scientific, Inc., Waltham, MA, USA). Following measurement of RNA concentration, reverse transcription was performed using a SuperScript RT kit (Takara Biotechnology Co., Ltd., Dalian, China) under the following conditions: $37^{\circ} \mathrm{C}$ for $15 \mathrm{~min}, 85^{\circ} \mathrm{C}$ for $5 \mathrm{sec}$, and then maintained at $4^{\circ} \mathrm{C}$. RT-PCR was performed in a $25 \mu \mathrm{l}$ reaction volume: $1 \mu \mathrm{l}$ diluted cDNA, $1 \mu \mathrm{l}$ sense primer $(10 \mathrm{pmol} / \mu \mathrm{l}), 1 \mu \mathrm{l}$ antisense primer $(10 \mathrm{pmol} / \mu \mathrm{l}), \mathrm{MgCl}_{2} 0.3 \mu \mathrm{l}$, $12.5 \mu 1$ Premix EX Taq II (2X), and 9.2 $\mu 1$ RNase-free $\mathrm{d}_{2} \mathrm{O}$. 
Following mixing, the cDNAs were synthesized under the following conditions: $94^{\circ} \mathrm{C}$ for $2 \mathrm{~min}, 94^{\circ} \mathrm{C}$ for $30 \mathrm{sec}, 60^{\circ} \mathrm{C}$ for $1 \mathrm{~min}$ at 40 cycles, $72^{\circ} \mathrm{C}$ for $30 \mathrm{sec}, 72^{\circ} \mathrm{C}$ for $10 \mathrm{~min}$, and $10^{\circ} \mathrm{C}$ for $10 \mathrm{~min}$ by PCR. The primers for gene amplification were synthesized by Shenggong Biological Engineering Technology and Services (Shanghai, China): Bax sense, 5'-ACAGATCAT GAAGACAGGGG-3' and antisense, 5'-CAAAGTAGAAGA GGGCAACC-3; Bcl-2 sense, 5'-TTCTCCTTCCAGCCTGAG AGC-3' and antisense, 5'-ATGACCCCACCGAACTCAAAG-3'; GAPDH sense, 5'-GGTGAAGGTCGGTGTGAACG-3' and antisense, 5'-CTCGCTCCTGGAAGATGGTG-3'. GAPDH was used as an internal control. The fidelity of all RT-PCR assays was assessed by $1.5 \%$ agarose gel electrophoresis containing $20 \mathrm{~g} / 1$ TAE buffer, and the relative gene expression levels were calculated relative to GAPDH.

Protein extraction and western blotting. Western blot analysis was executed to measure the protein levels of C5a, caspase- 3 and cleaved caspase-3. Briefly, the hepatic tissues were crushed, and the total protein was extracted from the cell lysate using T-PER ${ }^{\mathrm{TM}}$ Tissue Protein Extraction Reagent (cat. no. 78510; Thermo Fisher Scientific, Inc., Waltham, MA, USA), which was prepared with an inhibitor cocktail against proteases and phosphatases. Then the protein concentration in samples was measured using the Pierce ${ }^{\mathrm{TM}}$ BCA Protein Assay kit (cat. no. 23227; Thermo Fisher Scientific, Inc.). An equivalent amounts of protein $(80 \mu \mathrm{g})$ were separated SDS-PAGE on $15 \%$ gels and transferred to a polyvinylidene difluoride membrane at $4^{\circ} \mathrm{C}$. Following this, the membranes were blocked for $2 \mathrm{~h}$ at room temperature using Tris-buffered saline containing $0.1 \%$ Tween-20 and 5\% skimmed milk at room temperature. The membrane was then incubated at $4^{\circ} \mathrm{C}$ overnight with anti-C5a antibody (cat. no. ab194637; Abcam; 1:500), caspase-3 antibody (cat. no. ab13847; 1:100), cleaved caspase-3 antibody (cat. no. ab2302; 1:500), or anti-GAPDH antibody (cat. no. ab49822; 1:500; all Abcam). Subsequently, the membranes were incubated with the goat anti-mouse (cat. no. ab6789; $1: 5,000)$ and anti-rabbit secondary antibodies (cat. no. ab191866; 1:500; both Abcam) for $2 \mathrm{~h}$ at room temperature. Antibody binding was detected using an electrochemiluminescence detection kit to produce a chemiluminescence signal, which was captured on X-ray film. Relative protein expression levels were calculated relative to GAPDH.

Terminal deoxynucleotidyl transferase-mediated deoxyuridine triphosphate nick end labeling (TUNEL) assay. A TUNEL assay kit was used to detect hepatic cell apoptosis as recommended by the manufacturer (Roche Diagnostics, Basel, Switzerland). The number of apoptotic liver cells was counted in five random fields at magnification, x400 and five consecutive sections per liver tissue.

Statistical analysis. Quantitative data are expressed as the mean \pm standard deviation. Two groups of comparison were analyzed using the unpaired Student's t-test, and multigroup comparisons were performed by one-way analysis of variance) test with post hoc contrasts by the Student-Newman-Keuls method. Survival time curves were estimated using Kaplan-Meier analysis and compared using the stratified log-rank test. Data were analyzed using SPSS 22.0 for Windows
(IBM Corp., Armonk, NY, USA). P<0.05 (two-tailed) was considered to indicated a statistically significant difference.

\section{Results}

$C V F$ pretreatment improves the prognosis of rats with IRI. No rats in the control group died. The survival times were significantly prolonged in the high-dose and low-dose CVF groups (CVF1 and CVF2, respectively), compared with the IRI group (all $\mathrm{P}<0.01)$. However, no difference was observed between the CVF1 and CVF2 groups following IRI ( $>>0.05)$. Additionally, when CVF was administered at day 2 (CVF3 group) after IRI, the survival time was not improved compared with the IRI group ( $\mathrm{P}>0.05)$ (Fig. 1A).

CVF pretreatment attenuates the hepatic injury of rats with IRI. The low-dose CVF pretreatment (CVF2) was used for subsequent experiments because the survival times were not significantly difference between the high-dose and low-dose CVF groups following IRI ( $>0.05)$. In the CVF2 + IRI group, the levels of ALT and AST were significantly attenuated, compared with the IRI group at 3, 6, and $24 \mathrm{~h}$ (all $\mathrm{P}<0.05)$; however, the levels of ALT and AST in the CVF2 + IRI group remained significantly increased compared with the control group (all $\mathrm{P}<0.05)$ (Fig. 1B and $\mathrm{C}$ ).

Furthermore, the H\&E staining results of the hepatic tissues of the control, IRI, and CVF2 + IRI groups (24 h) are presented in Fig. 1D. No hepatic cell injury was detected in the control group. However, the IRI group exhibited albuminoid and vacuolar degeneration, eosinophilic variants, spotty liver necrosis, inflammatory cell infiltration and Kupffer cell proliferation; while only focal eosinophilic variants of hepatic cells were observed in the CVF2 + IRI group.

CVF pretreatment results in complement and membrane attack complex depletion. To verify that CVF pretreatment induced complement and membrane attack complex depletion, plasma complement $\mathrm{CH} 50$ and $\mathrm{C} 5 \mathrm{~b}-9$ activities were determined in the control, IRI and CVF2 + IRI groups. The ELISA results demonstrated that IRI significantly increased complement $\mathrm{CH} 50$ and $\mathrm{C} 5 \mathrm{~b}-9$ expression, and the expression levels reached a peak at $1 \mathrm{~h}$ after IRI initiation, compared with the control group (all $\mathrm{P}<0.05$ ) (Fig. $2 \mathrm{~A}$ and $\mathrm{B}$ ). When IRI rats received CVF2 pretreatment, the $\mathrm{CH} 50$ and $\mathrm{C} 5 \mathrm{~b}-9$ activities were significantly decreased compared with those of the rats in the IRI group within $24 \mathrm{~h}$ (all $\mathrm{P}<0.05$ ) (Fig. $2 \mathrm{~A}$ and B); however, there was no significant difference in CH50 and C5b-9 levels between the IRI group and the CVF2 + IRI group at $>24 \mathrm{~h}$.

Additionally, immunohistochemistry assay demonstrated that the level of C5b-9 deposition was significantly reduced in the CVF2 + IRI group at 3,6, and $24 \mathrm{~h}$, compared with the IRI group. However, the level of C5b-9 deposition was decreased in the CVF2 + IRI group compared with IRI rats; however, the C5b-9 deposition remained significantly higher than the control group (all $\mathrm{P}<0.05$ ) (Fig. 2C).

CVF pretreatment reduces the release of inflammatory mediators. The ELISA results demonstrated that TNF- $\alpha$ and IL-1 $\beta$ release was significantly increased in the IRI group compared 


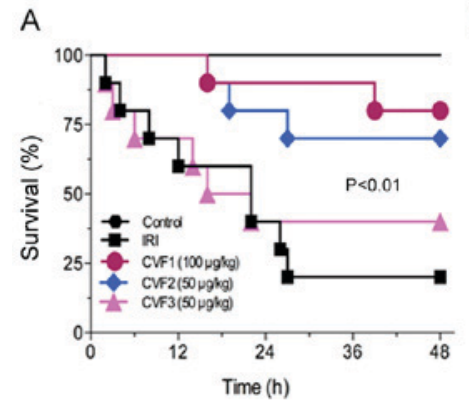

D

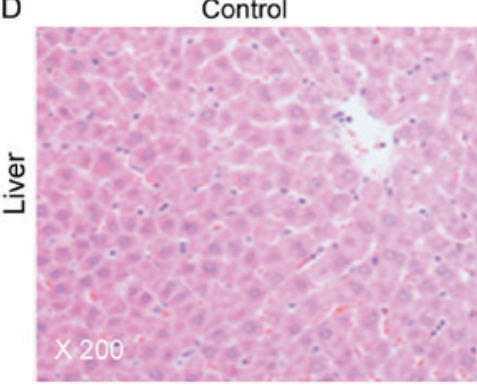

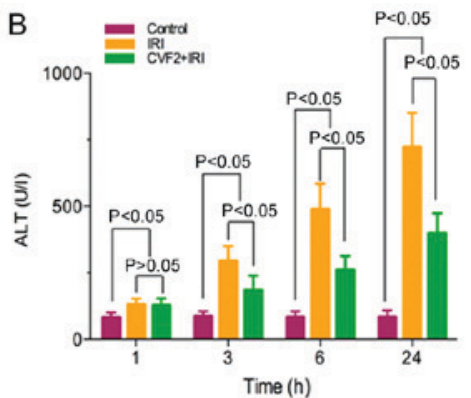

IRI

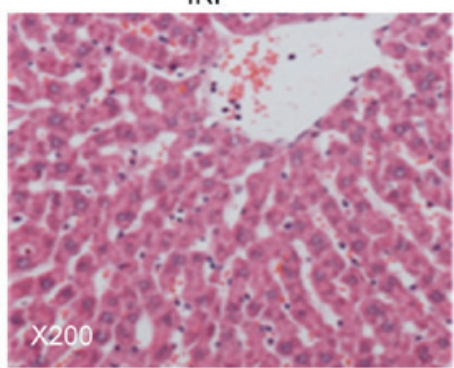

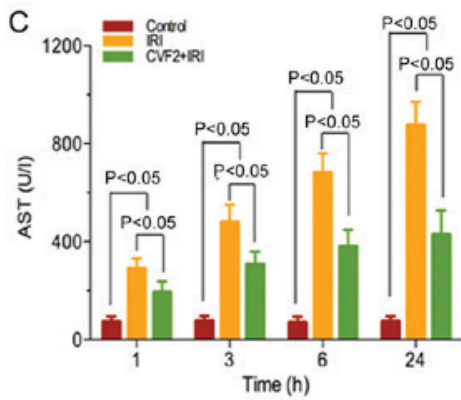

CVF2+IRI

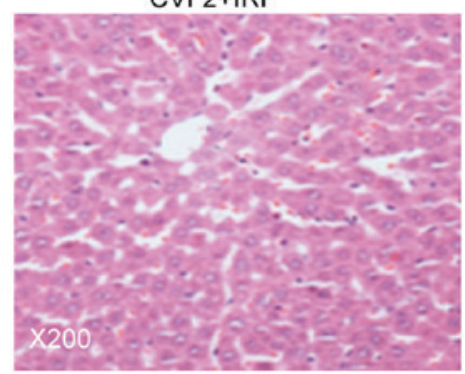

Figure 1. CVF pretreatment improves the prognosis and attenuates hepatic injury of rats with IRI. (A) Survival times under different CVF treatment doses and schedules for rats with IRI were evaluated (10 rats per group). The liver function markers (B) ALT and (C) AST were assessed using an ELISA at different times following IRI initiation. (D) Pathological changes in the liver at $24 \mathrm{~h}$ among the control, IRI and CVF2 + IRI groups. Hepatic tissues stained with hematoxylin and eosin, and observed under a light microscope (magnification, x200). IRI, ischemia-reperfusion injury; CVF, cobra venom factor; ALT, alanine transaminase; AST, aspartate transaminase.
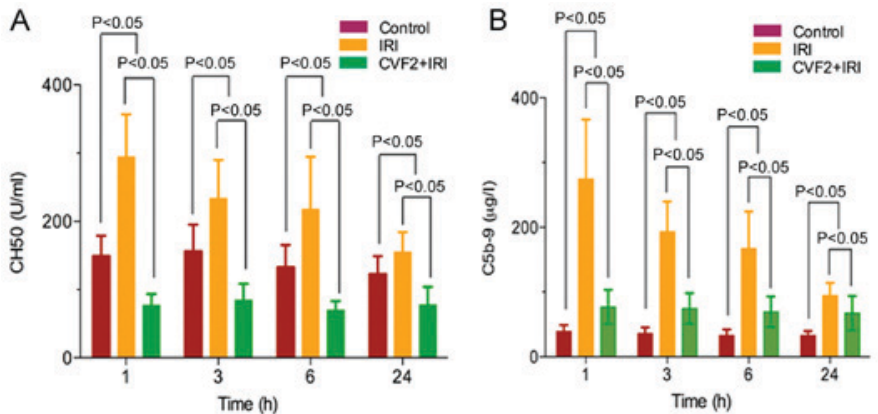

C
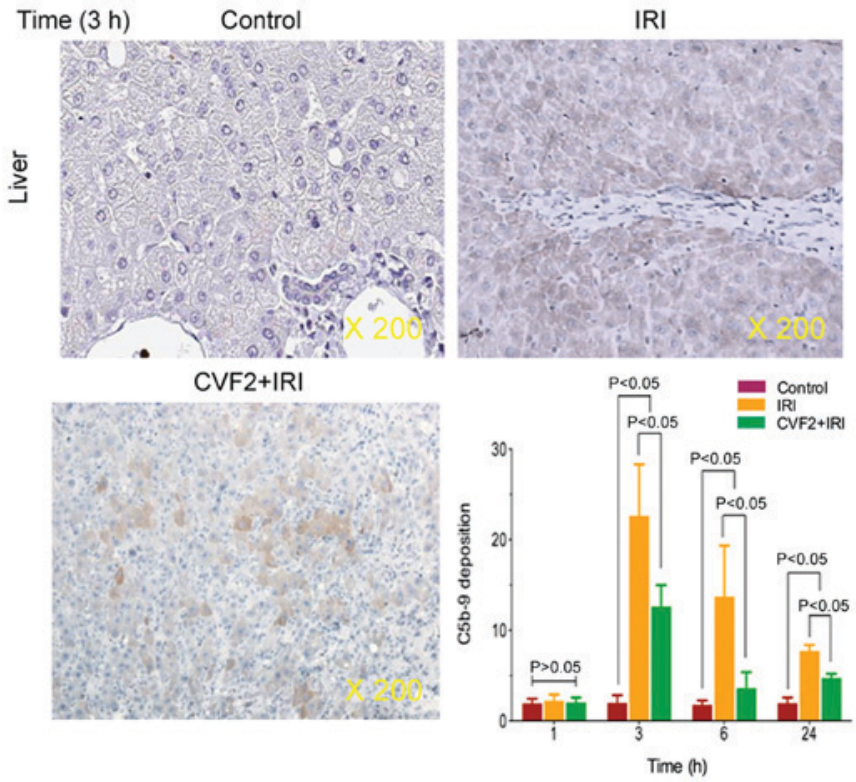

Figure 2. CVF pretreatment resulted in complement and membrane attack complex depletion. (A) Complement CH50 and (B) membrane attack complex C5b-9 were assessed using ELISAs within $24 \mathrm{~h}$ after IRI initiation. (C) C5b-9 deposition in liver tissues of the control, IRI and CVF2 + IRI groups were assessed by immunohistochemical staining. Data are representative at $3 \mathrm{~h}$ (magnification, x200). IRI, ischemia-reperfusion injury; CVF, cobra venom factor; CH50, total hemolytic complement. 
A
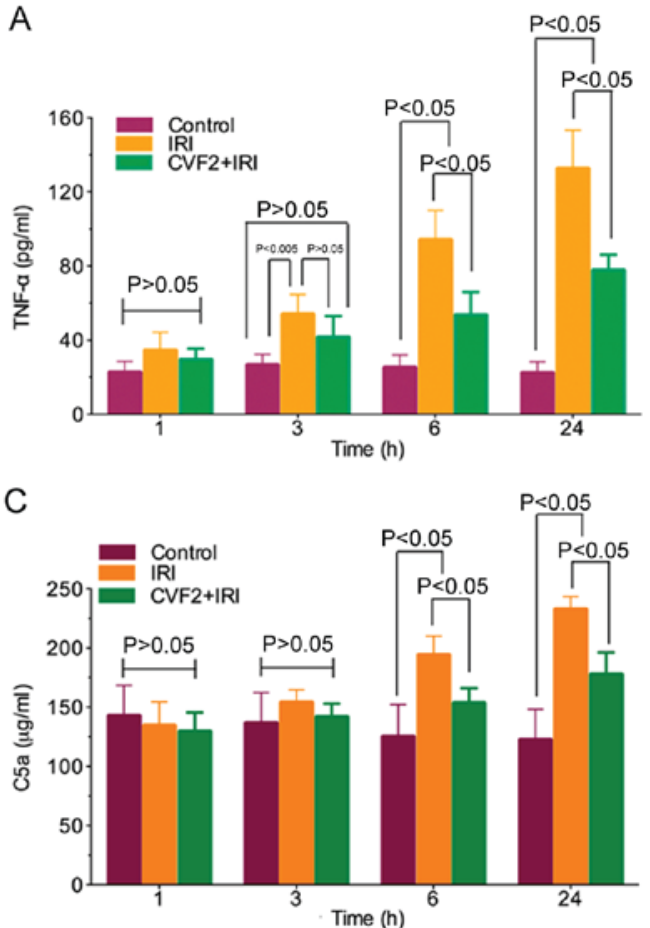

B

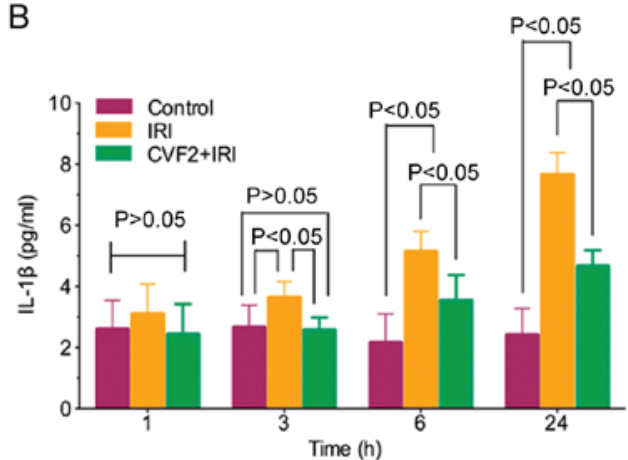

D

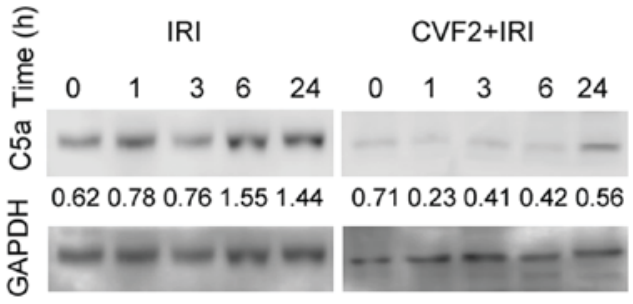

Figure 3. CVF pretreatment reduces the release of inflammatory mediators. Inflammatory mediators (A) TNF- $\alpha$ and (B) IL-1 $\beta$, and (C) anaphylatoxin C5a were assessed using ELISAs at different times following IRI initiation. (D) Expression of anaphylatoxin C5a protein was evaluated by western blotting analysis in liver tissues at different times after IRI initiation, and the protein levels were calculated relative to GAPDH. IRI, ischemia-reperfusion injury; CVF, cobra venom factor; TNF- $\alpha$, tumor necrosis factor- $\alpha$; IL-1 $1 \beta$, interleukin- $1 \beta$.

with the control group (all $\mathrm{P}<0.05$ ). Following $\mathrm{CVF} 2$ pretreatment, the levels of inflammatory mediator release were significantly reduced at 3,6 , and $24 \mathrm{~h}($ all $\mathrm{P}<0.05)$ (Fig. 3A and $\mathrm{B})$.

Activation of the complement system generates anaphylatoxin C5a. In the IRI group, C5a expression was significantly increased compared with the control group at 6 and $24 \mathrm{~h}$ (all $\mathrm{P}<0.05$ ). By contrast, pretreatment with CVF2 attenuated the elevation of C5a expression in the IRI group at 6 and $24 \mathrm{~h}$ $(\mathrm{P}<0.05)$ (Fig. 3C). Consistent with the ELISA outcomes, the $\mathrm{C} 5 \mathrm{a}$ protein level in the liver tissues within $24 \mathrm{~h}$ were also significantly reduced by CVF2 pretreatment, compared with the IRI group, according to western blot analysis (Fig. 3D).

CVF pretreatment alleviates the apoptosis of liver cells. The levels of apoptotic hepatic cells were observed using a TUNEL assay. No apoptotic cells were detected in the control group. However, the IRI rats exhibited a marked increase in apoptotic liver cells; whereas CVF2 pretreatment significantly reduced the hepatic cell apoptosis at 3, 6 and $24 \mathrm{~h}$ (all $\mathrm{P}<0.05$ ) (Fig. 4A).

Compared with the IRI group, the Bax expression and $\mathrm{Bax} / \mathrm{Bcl}-2$ ratio were significantly lower in the CVF2 group within $24 \mathrm{~h}$ (all $\mathrm{P}<0.05$; Fig. $4 \mathrm{~B}$ and $\mathrm{C}$ ). Consistent with the reduced $\mathrm{Bax}$ expression and $\mathrm{Bax} / \mathrm{Bcl}-2$ ratio, pretreatment with CVF2 markedly attenuated the elevation of cleaved caspase- 3 expression within $24 \mathrm{~h}$ (Fig. 4D).

\section{Discussion}

IRI can induce acute hepatic injury, which is associated with high mortality rates. The liver is subjected to IRI in several medical conditions, including hepatic resection and liver transplantation. Increasing evidence has demonstrated that complement activation is required for IRI-induced hepatic damage (5-8), and CVF can deplete the complements of the component system (9-11). The current study investigated the effect and intrinsic mechanism of CVF pretreatment on acute hepatic injury induced by IRI in rats, and the results indicated that CVF pretreatment ameliorates IRI-induced acute hepatic injury. However, further studies are required to determine whether this therapy could be a potential agent for the treatment of IRI injuries in clinical settings.

In the present study, the survival time of rats was significantly prolonged in the high-dose and low-dose CVF pretreatment groups (CVF1 and CVF2 groups, respectively), compared with the IRI group. CVF administration at day 2 (CVF3 group) after IRI did not improve the survival time, compared with the IRI group. These results suggested the involvement of complement activation at the early stage, not the late stage, following IRI. In addition, either high-dose or low-dose CVF pretreatment improved the survival time of rats with IRI.

In the current study, when IRI was initiated, the liver function significantly deteriorated compared to the control group; while CVF pretreatment significantly improved the liver function. However, the exact intrinsic mechanism of CVF pretreatment in attenuating hepatic injury following IRI is remains unknown. In the present study, the CH50 and C5b-9 levels were significantly increased, and their expression levels reached a peak at $1 \mathrm{~h}$ after IRI initiation. These results were in agreement with previous studies (14), suggesting that CVF pretreatment results in complement and 
A
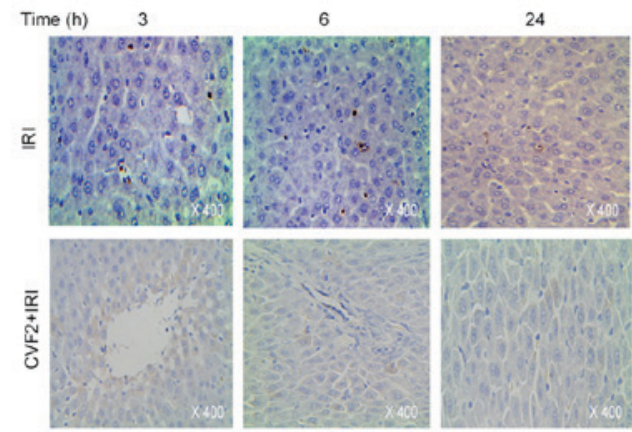

B
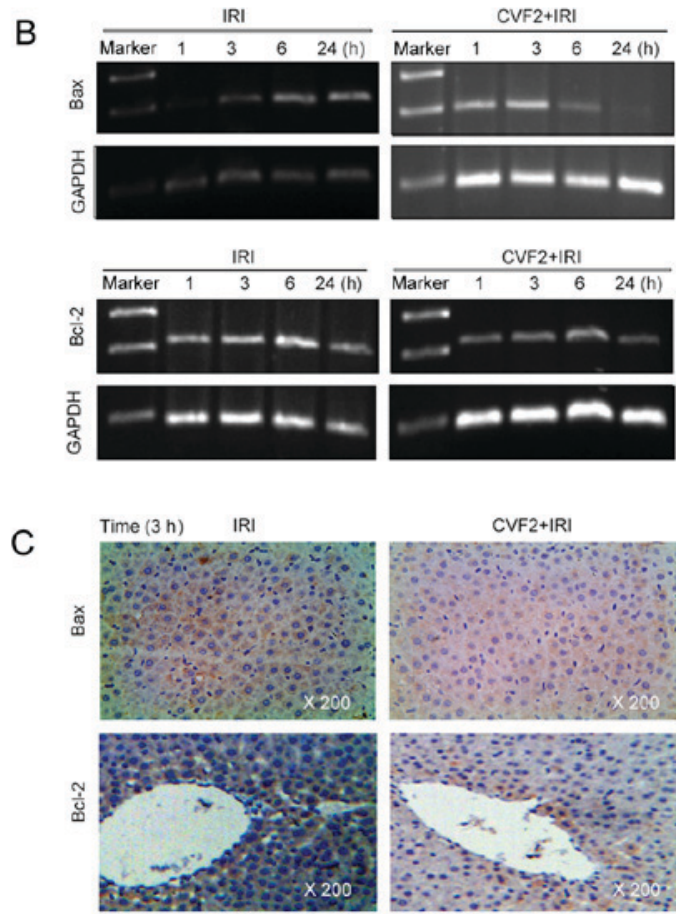
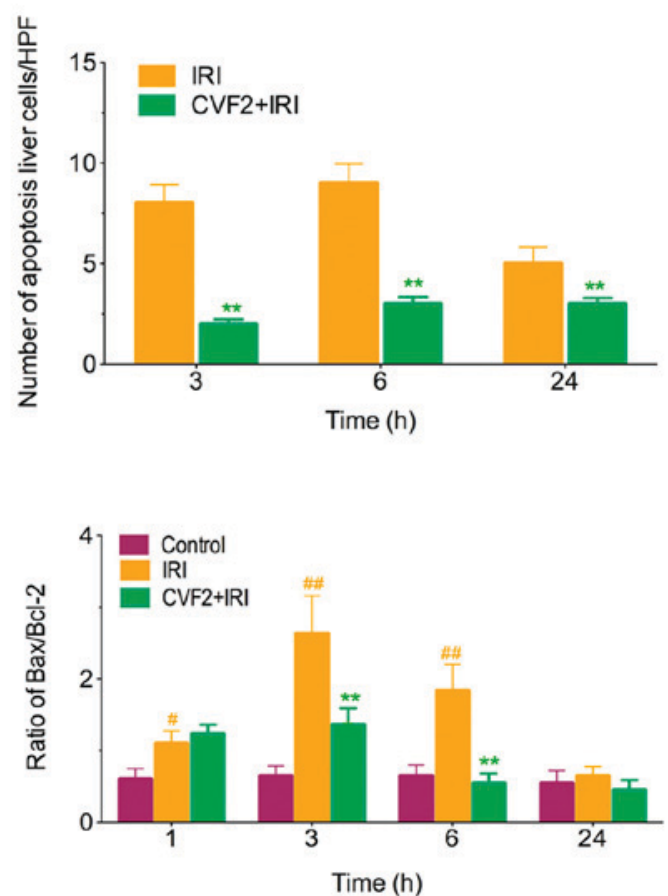

D
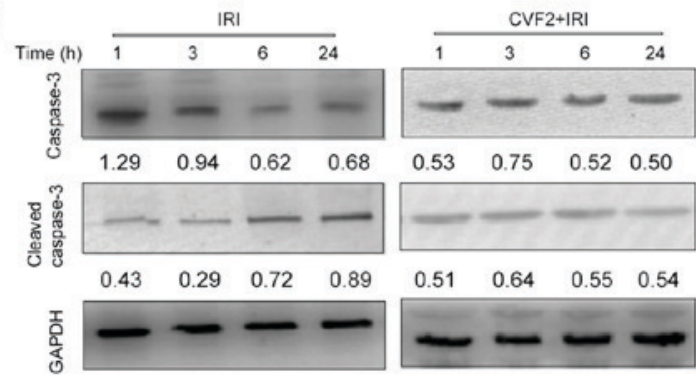

Figure 4. CVF pretreatment alleviates liver cell apoptosis. (A) The liver cell apoptosis levels were determined by a TUNEL assay (x400) at different times after IRI initiation. The proapoptotic protein Bax, inhibition of apoptotic protein Bcl-2, and the ratio of Bax/Bcl-2 were assessed by (B) RT-PCR and (C) immunohistochemical (x200) assays at different times after IRI initiation. (D) The protein expression levels of caspase-3 and cleaved caspase-3 were evaluated by western blotting assays of liver tissues at different times after IRI initiation, and the protein levels were calculated relative to GAPDH. ${ }^{* *} \mathrm{P}<0.01$ vs. the IRI group; ${ }^{\#} \mathrm{P}<0.05$ vs. the control group; ${ }^{\# \#} \mathrm{P}<0.01$ vs. the control group. IRI, ischemia-reperfusion injury; CVF, cobra venom factor; HPF, high-powered field; Bax, $\mathrm{Bcl}-2$ associated $\mathrm{X}$, apoptosis regulator; $\mathrm{Bcl}-2, \mathrm{Bcl}-2$ apoptosis regulator.

membrane attack complex depletion, and that complement activation is involved in the pathogenesis of hepatic injury following IRI.

Notably, when the IRI rats received CVF pretreatment, the CH50 and C5b-9 activities were significantly decreased within $24 \mathrm{~h}$, but not at $>24 \mathrm{~h}$, compared with the rats in the IRI group. These results suggested that systemic complement activity was gradually restored. The return of complement activity may have been due to the development of antibodies against CVF or due to complement regeneration in the liver. Therefore, the window of time available for therapeutic intervention to block complement-mediated events in the post-IRI period may be important.

The mechanism by which complement activity contributes to hepatic injury remains unresolved. Figueroa et al (13) has demonstrated that CVF administration may allow accommodation to take place by impairing the ability of anaphylatoxin C5a to form. Given that the release of inflammatory mediators such as TNF- $\alpha$ and IL-1 $\beta$ was significantly decreased in the IRI rats receiving CVF pretreatment, inflammatory mediator release is potentially actively involved in complement activation (12). In addition, considering that the number of apoptotic of liver cells was significantly increased in the rats with IRI, it is likely that apoptosis may participate in complement activation by mediating caspase activity, as reported in Gorsuch et al study (5).

In conclusion, CVF pretreatment ameliorated IRI-induced acute hepatic injury in rats. The results of this study were consistent with those of previous research (12-15) may result in novel therapies based on the strategy of complement inhibition to ameliorate multiple organ injury resulting from IRI. Several clinically applicable complement inhibitors are in development or in preclinical trials for various disorders (19). Continued research may yield data justifying clinical trials of one or more complement inhibitors administered during or shortly after events characterized by IRI. 


\section{Acknowledgements}

Not applicable.

\section{Funding}

The present study was supported in part by the Foundation of Tianjin Heath and Family Planning Commission (grant nos. 14KG101 and 2014KR07), and the National Clinical Key Specially Project Foundation of the Ministry of Health in China (grant no. 2011-873) to Professor Yong-Qiang Wang.

\section{Availability of data and materials}

The analyzed data sets generated during the study are available from the corresponding author on reasonable request.

\section{Authors' contributions}

BW carried out conception and design, performed experimental study, collection and assembly of data, data analysis and interpretation, manuscript writing. HX, JL and HMG performed the experimental study, collection and assembly of data, data analysis and interpretation. YHX, ZL and HJL performed collection and assembly of data, data analysis and interpretation. YQW and SHC carried out conception and design, collection and assembly of data, data analysis and interpretation, manuscript writing. All authors read and approved the final manuscript.

\section{Ethics approval and consent to participate}

All experimental procedures were performed according to the Animal Care Committee guidelines, and the experimental protocol was approved by the Ethics Committee of Tianjin First Center Hospital (approval no. 2015013Z).

\section{Patient consent for publication}

Not applicable.

\section{Competing interests}

The authors declare that they have no competing interests.

\section{References}

1. Cannistrà $M$, Ruggiero $M$, Zullo $A$, Gallelli $G$, Serafini $S$, Maria M, Naso A, Grande R, Serra R and Nardo B: Hepatic ischemia reperfusion injury: A systematic review of literature and the role of current drugs and biomarkers. Int J Surg 33 (Suppl 1): S57-S70, 2016.
2. Peralta C, Jiménez-Castro MB and Gracia-Sancho J: Hepatic ischemia and reperfusion injury: Effects on the liver sinusoidal milieu. J Hepatol 59: 1094-1106, 2013.

3. Abe Y, Hines IN, Zibari G, Pavlick K, Gray L, Kitagawa Y and Grisham MB: Mouse model of liver ischemia and reperfusion injury: Method for studying reactive oxygen and nitrogen metabolites in vivo. Free Radic Biol Med 46: 1-7, 2009.

4. Pantazi E, Bejaoui M, Folch-Puy E, Adam R and Roselló-Catafau J: Advances in treatment strategies for ischemia reperfusion injury. Expert Opin Pharmacother 17: 169-179, 2016.

5. Gorsuch WB, Chrysanthou E, Schwaeble WJ and Stahl GL: The complement system in ischemia-reperfusion injuries. Immunobiology 217: 1026-1033, 2012.

6. Bajic G, Degn SE, Thiel S and Andersen GR: Complement activation, regulation, and molecular basis for complement-related diseases. EMBO J 34: 2735-2757, 2015.

7. Melis JP, Strumane K, Ruuls SR, Beurskens FJ, Schuurman J and Parren PW: Complement in therapy and disease: Regulating the complement system with antibody-based therapeutics. Mol Immunol 67: 117-130, 2015 .

8. Krishnan V, Ponnuraj K, Xu Y, Macon K, Volanakis JE and Narayana SV: The crystal structure of cobra venom factor, a cofactor for C3- and C5-convertase CVFBb. Structure 17: 611-619, 2009.

9. Vogel CW and Fritzinger DC: Cobra venom factor: Structure, function, and humanization for therapeutic complement depletion. Toxicon 56: 1198-1222, 2010.

10. Vogel CW, Finnegan PW and Fritzinger DC: Humanized cobra venom factor: Structure, activity, and therapeutic efficacy in preclinical disease models. Mol Immunol 61: 191-203, 2014.

11. Mao YF, Yu QH, Zheng XF, Liu K, Liang WQ, Wang YW, Deng XW and Jiang L: Pre-treatment with cobra venom factor alleviates acute lung injury induced by intestinal ischemia-reperfusion in rats. Eur Rev Med Pharmacol Sci 17: 2207-2217, 2013.

12. Gorsuch WB, Guikema BJ, Fritzinger DC, Vogel CW and Stahl GL: Humanized cobra venom factor decreases myocardial ischemia-reperfusion injury. Mol Immunol 47: 506-510, 2009.

13. Figueroa E, Gordon LE, Feldhoff PW and Lassiter HA: The administration of cobra venom factor reduces post-ischemic cerebra injury in adult and neonatal rats. Neurosci Lett 380: 48-53, 2005.

14. Vogel CW, Fritzinger DC, Gorsuch WB and Stahl GL: Complement depletion with humanized cobra venom factor: Efficacy in preclinical models of vascular disease. Thromb Haemost 113: 548-552, 2015.

15. Sun QY, Chen G, Guo H, Chen S, Wang WY and Xiong YL: Prolonged cardiac xenograft survival in guinea pig-to-rat model by a highly active cobra venom factor. Toxicon 42: $257-262$, 2003.

16. Cui YL, Qiu LH, Zhou SY, Li LF, Qian ZZ, Liu XM, Zhang HL, Ren XB and Wang YQ: Necroptosis as a potential therapeutic target in multiple organ dysfunction syndrome. Oncotarget 8: 56980-56990, 2017.

17. Silverman J and Muir WW III: A review of laboratory animal anesthesia with chloral hydrate and chloralose. Lab Anim Sci 43: 210-216, 1993.

18. Zimpfer M, Silt SP and Vatner SF: Effect of anesthesia on the canine carotid chemoreceptor reflex. Circ Res 48: 400-406, 1981.

19. Bahde R and Spiegel HU: Hepatic ischemia-reperfusion injury from bench to beside. Br J Surg 97: 1461-1475, 2010.

This work is licensed under a Creative Commons Attribution-NonCommercial-NoDerivatives 4.0 International (CC BY-NC-ND 4.0) License. 